\title{
A Study of the Yosemite Sequence Used as a Test Sequence for Estimation of Optical Flow
}

\author{
Ivar Austvoll \\ Signal and Image Processing Group, \\ Department of Electrical Engineering and Computer Science, \\ University of Stavanger, N-4036 Stavanger, Norway \\ Ivar.Austvoll@uis.no
}

\begin{abstract}
Since the publication of the comparative study done by Barron et al. on optical flow estimation, a race was started to achieve more and more accurate and dense velocity fields. For comparison a few synthetic image sequences has been used. The most complex of these is the Yosemite Flying sequence that contains both a diverging field, occlusion and multiple motions at the horizon. About 10 years ago it was suggested to remove the sky region because the correct flow used in earlier work was not found to be the real ground truth for this region. In this paper we present a study of the sky region in this test sequence, and discuss its usefulness for evaluation of optical flow estimation.
\end{abstract}

\section{Introduction}

It took about 30 years from the introduction of the conception of optical flow by Gibson [1] till the breaking through of algorithms for estimation of optical flow $[2,3]$. Since then we have had a steady growth in the number of new approaches for optical flow estimation. An important event was the comparative study done by Barron et al. [5, 6, that started a competition towards better accuracy for a few synthetic image sequences. Here it is preposterous to mention all the participants in this struggle towards better results. We have chosen some of the best results reported and visualized the latest developments in this area graphically. Names will be missing, but we hope to present a representative choice of techniques and their results.

The focus in this paper is to demonstrate some of the peculiarities of the sky region for the Yosemite sequence and look at the consequences this has for the use as a test sequence.

In the next section we give some information on the Yosemite sequence. Then we discuss the error measure used for comparison of the different techniques, and

\footnotetext{
${ }^{1}$ We have here not mentioned the efforts in motion estimation connected to image sequence processing. Some of the earliest results in this field are from Limb and Murphy [4.
} 
we present some of the results reported since 1980. We continue with some simple experiments on the sky region, and finally we give a discussion of the results and conclude our work.

\section{The Yosemite Sequence}

The Yosemite sequence was created by Lynn Quam at SRI [5]. This sequence is based on an arial image of the Yosemite valley and synthetic generated by flying through the valley. In the sky region clouds are generated based on fractal textures. The ground truth for the motion field is known for the valley part, but it is questionable if the ground truth used for the sky region is correct 7 . According to Black in [7] there is no ground truth for the cloud motion. The cloud pattern is fractal and undergoing Brownian motion. The assumption of brightness constancy does not hold for the clouds. So reporting errors in the cloud region is completely meaningless. According to our view this statement is a bit too strong and only partly true.

The first image frame in the Yosemite sequence is shown in Figure 1, left. To analyze the situation in the sky region we will use a simple approach, where space-time-slices, ST-slices [8], are extracted for the rows (The motion is purely horizontal in the sky region.). Examples are found in Figure 1, right. The uppermost ST-slice is from the original signal, the middle is the phase output from a directional filter and the nethermost is the magnitude output from the directional filter. It is well known, and also illustrated in these ST-slices, that the component velocity in the direction of the ST-slice is given by the orientation of the ST-signal. To estimate the component velocity we can use a method for orientation estimation. This problem is discussed in [9], where a new set of quadrature filters, loglets is introduced. As stated in [9] the estimation of orientation and estimation of velocity is identical when the signal
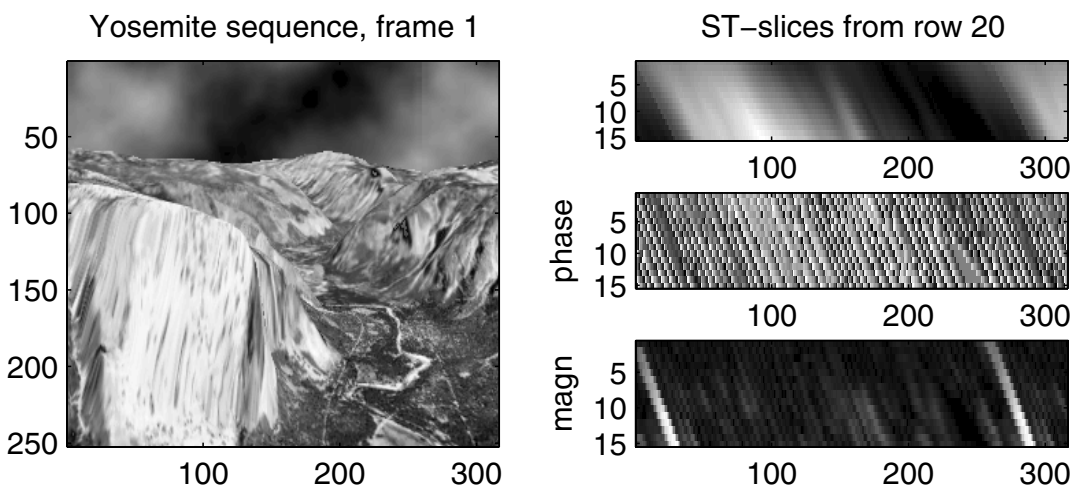

Fig. 1. Left: first image of the Yosemite sequence. Right: space-time-slices, original signal, phase and magnitude from directional filter 
is bandlimited. In our work we have used a quadrature filter bank based on the discrete Prolate Spheroidal sequence (DPSS) (equivalent to the Kaiserwindow) [10,11]. To bandlimit the signal we used the scale space filter suggested in [12].

\subsection{Error Measure}

In most published work on optical flow estimation after Barron et al. and their comparative study, the angular error measure introduced by Fleet and Jepson [13 has been used. The argument for introducing this error measure was that the length of the vector error $\left(\mathbf{v}-\mathbf{v}^{\text {corr }}\right)$, where $\mathbf{v}$ is the measured velocity and $\mathbf{v}^{\text {corr }}$ the ground truth, is not useful as a measure of error because it depends on the speed (speed $=$ magnitude of the velocity vector). To compute this measure we use the $3 \mathrm{D}$ space-time velocity vectors, $\boldsymbol{v}=\left[\begin{array}{ll}\mathbf{v} & 1\end{array}\right]^{T}$ and $\boldsymbol{v}^{\text {corr }}=\left[\begin{array}{ll}\mathbf{v}^{\text {corr }} & 1\end{array}\right]^{T}$ with lengths, $\|\boldsymbol{v}\|$ and $\left\|\boldsymbol{v}^{\text {corr }}\right\|$ respectively. The angular error measure is then given by:
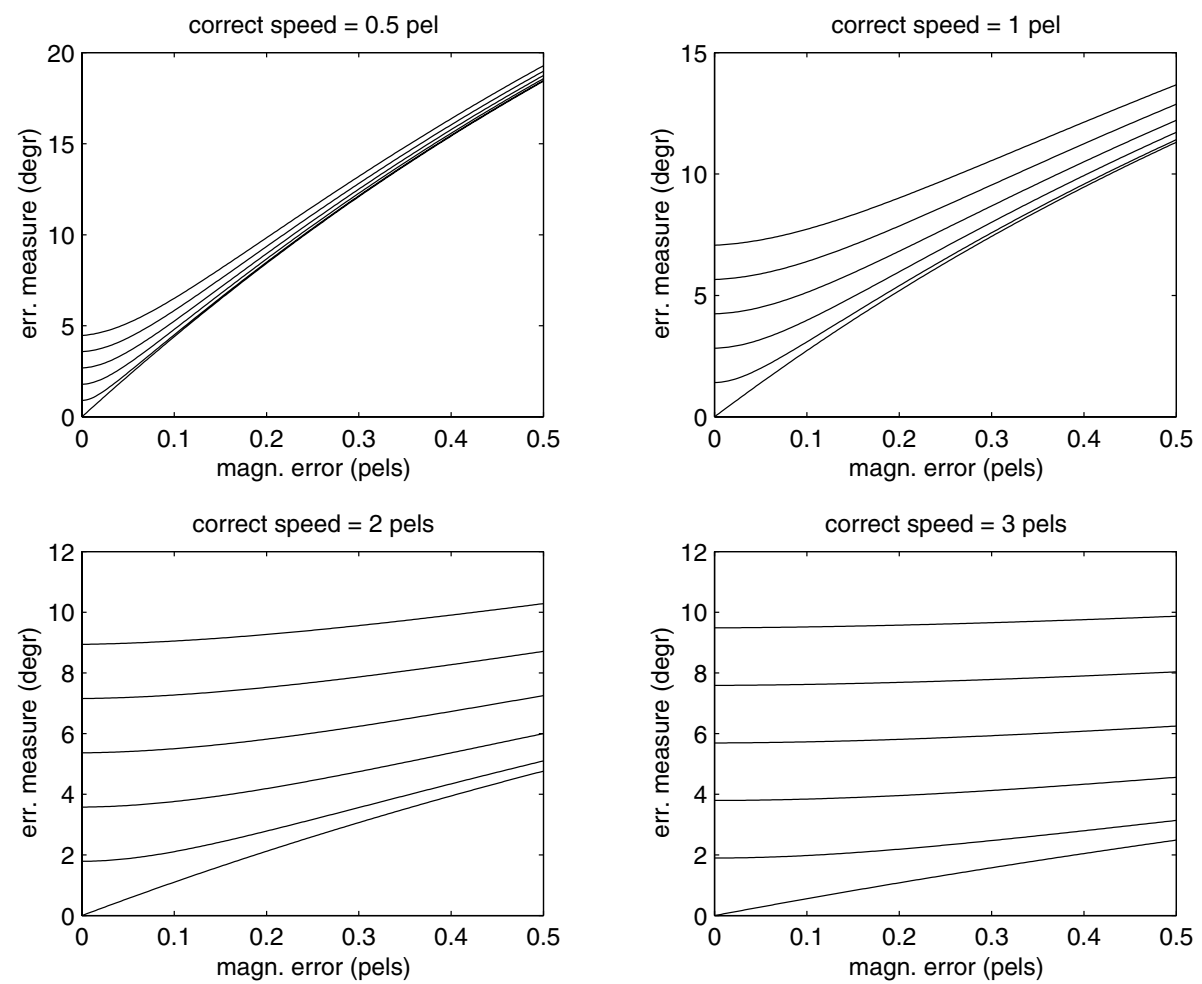

Fig. 2. Angular error measure as a function of magnitude error, $v_{e r r}$, for absolute angular errors, $\alpha_{e}$, of $0,2,4,8,10$ degrees, at different correct speeds, computed from equation (11) 


$$
\varphi_{e}=\arccos \left(\frac{\boldsymbol{v}^{T} \boldsymbol{v}^{\text {corr }}}{\|\boldsymbol{v}\|\left\|\boldsymbol{v}^{\text {corr }}\right\|}\right) .
$$

For the estimated velocity vectors there can be an error in direction, angular error $\alpha_{e}=\operatorname{ang}(\mathbf{v})-\operatorname{ang}\left(\mathbf{v}^{\text {corr }}\right)$, and in the length of the vector, magnitude error $v_{\text {err }}=|\mathbf{v}|-\left|\mathbf{v}^{\text {corr }}\right|$. Some of the properties of this error measure can be seen from Figure 2 .

\subsection{Error Statistics}

Succeeding the work of Barron et al. 5] a bunch of competing methods for estimation of optical flow has been published. We will here only focus on the average error and the standard deviation found for the Yosemite sequence. Two versions of the results exist, with clouds and without clouds. From about 1994 some researchers followed the attitude of Black [7] and skipped the clouds.

For the Yosemite sequence including clouds, error measures is shown in Figure 3, left. Here we have only included published results where the density is $100 \%$ and have chosen the best in each time slot. The results without clouds is
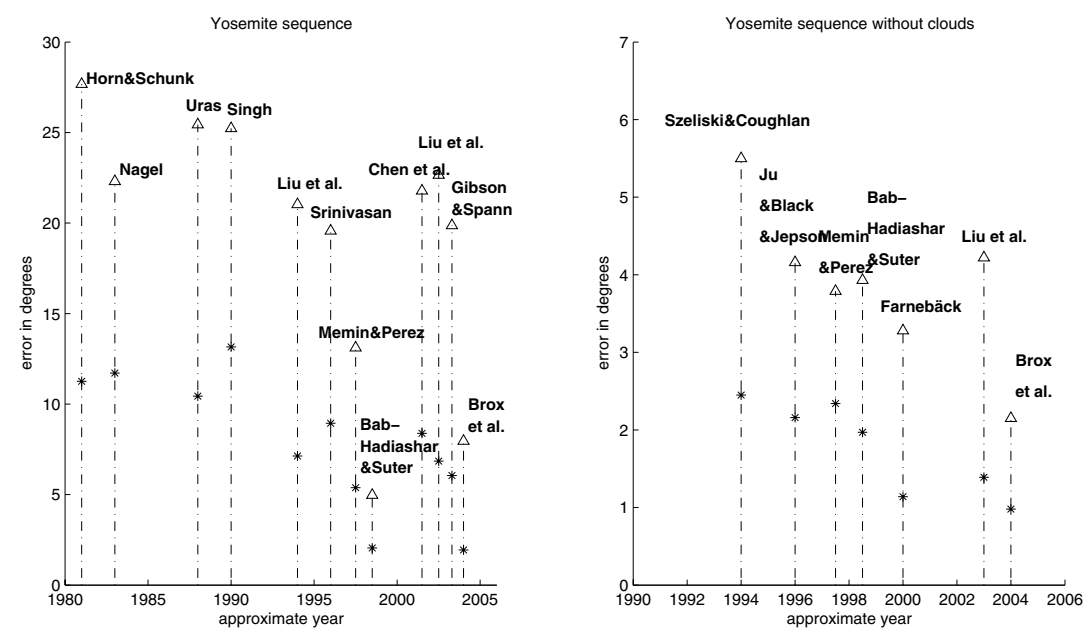

Fig. 3. History of error measures for the Yosemite sequence. The asterisks is placed at the average error while the arrows indicates the standard deviation

shown in Figure 3, right. Here only results from about 1994 are given. We have excluded the newly published results in 14 because the numbers seem not to be reasonable 2. The best results sofar is from Brox et al. [15] with less than

${ }^{2}$ The velocity arrows in Figure 1c) in the article does not correspond to the average error of 0.2 degrees that is given in the Table I for comparison with other methods. The published accuracy is almost an order of magnitude better than other methods! The reason is probably that a different error measure has been used. 
2 degrees average error with clouds, and less than 1 without clouds. Very close is the work of Bab-Hadiashar and Suter 16. For the Yosemite sequence with clouds these results stands out, especially with much better standard deviation. For the sequence without clouds the results are more even. In addition to the former, Liu et al. 17, Farnebäck 18 among others, have reported nice results. The best results seem to come from differential methods including multiscale approaches and both global and local smoothing [15] and methods based on robust statistics [16, 19, 20. Orientation tensors combined with affine models [18, 17] is another approach with good results. Reasonable results is also achieved with a wavelet based method [21].

From the results of the average angular error the relative contribution of angular and magnitude error it is not known. This can be of importance for practical applications. From Figure 2 we can deduce some conclusions. If the correct velocity is 2 pels (picture elements, pixels) the average magnitude error is less than about 0.2 pels in the worst case (no angular error) and the average angular error is less than 2 degrees when the average error is less than 2 degrees. This is the case in the sky region.

\section{$3 \quad$ Experiments}

The aim of this work has not been to give prominence to a special technique for estimation of optical flow. We only want to demonstrate the relative ability to estimate motion in the sky region. For our experiments we have therefore used a method at our hand, available from earlier work, with some smaller modifications. The accuracy of the results is therefore also moderate.

\subsection{Velocity Estimation Method}

The ST-slices are smoothed by a scale-space filter and each row is filtered by a 1D one-sided complex bandpass filter. The method is a simplified version of the one described in [10. The output is a complex ST-slice. The component velocity in the direction of the spatial axis for the ST-slice (in our case horizontal) can be computed from the direction of the pattern in the ST-slice, either from the original signal, the smoothed ST-slice or the phase from the filtered ST-slice. This can be done by a structure tensor 22, 8, by a set of quadrature filters 22, 10, or any suitable method for estimation of directional patterns. We have chosen the approach in [10, used only one scale and computed the component velocity in the horizontal direction.

\subsection{Results}

To demonstrate the problems introduced in the sky region we have computed the horizontal component of the velocity (the vertical component is zero in this region) for rows. From visual inspection of the ST-slices, see Figure 1, right, it is obvious that the velocity is about 2 pels from left to right. From the original signal we can also confirm that the brightness constancy is violated, but the 

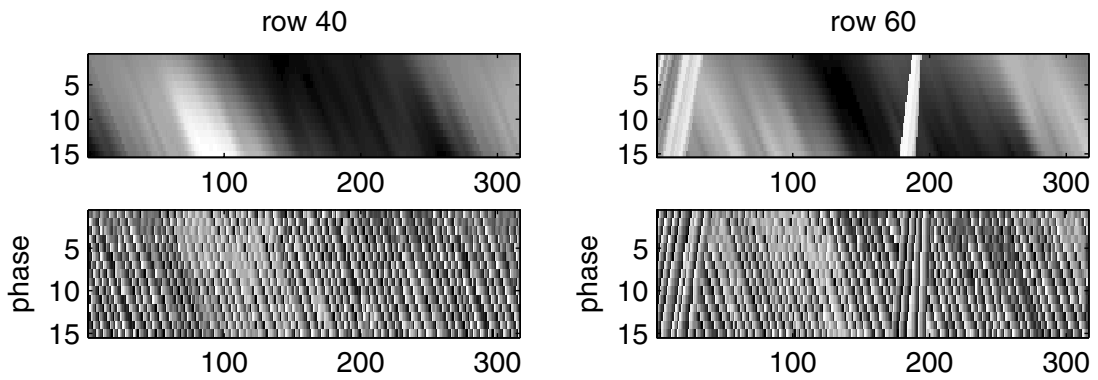

Fig. 4. ST-slices

space-time tracks have the expected direction. The signal from the directional filter is more conclusive. The phase has a very distinct direction in space-time, visually, when we use a global view. For local regions the case is not so obvious, with many dislocations from the main direction. The ST-slices for two more rows is found in Figure 4. The left figures show ST-slices at row number 40. The motion in a direction to the right is obvious. The right part of this figure show ST-slices from row number 60. This is just at the horizon.
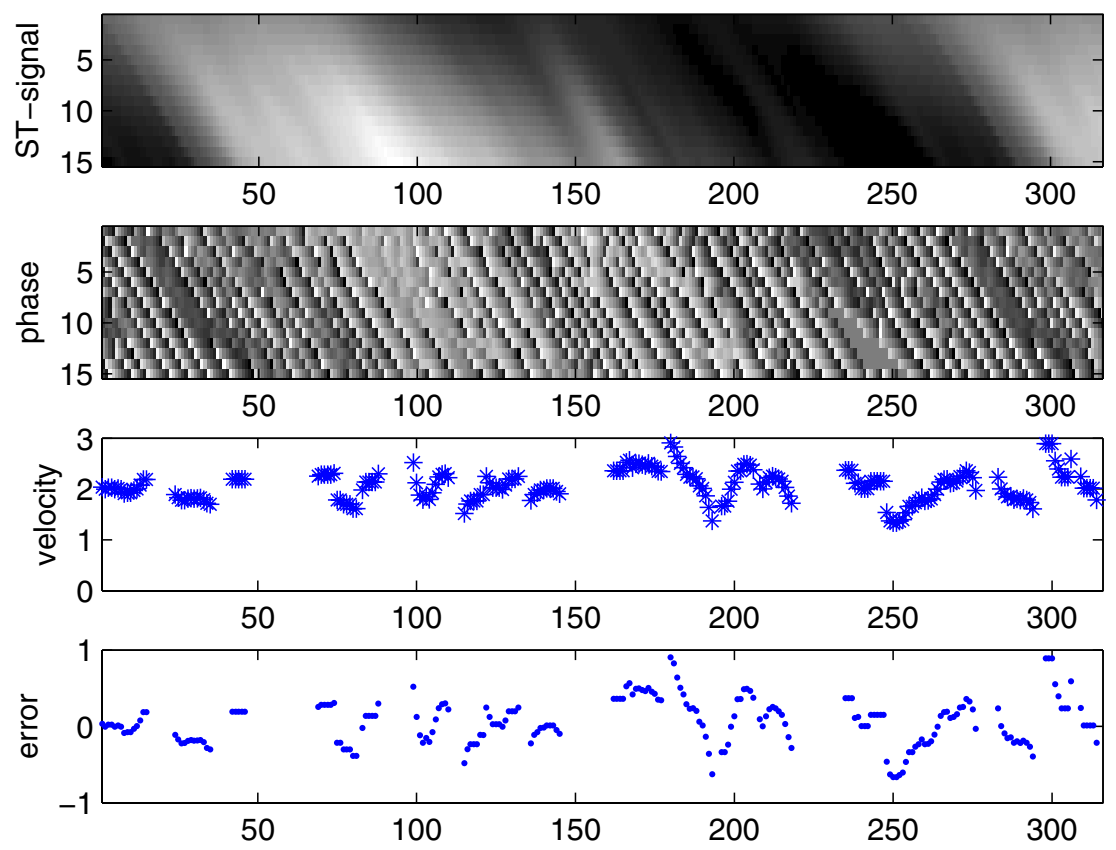

Fig. 5. ST-signals, original and phase for row number 20, upper figures. Estimated magnitude velocity for row number 20 at frame number 7 and corresponding errors, lower plots 

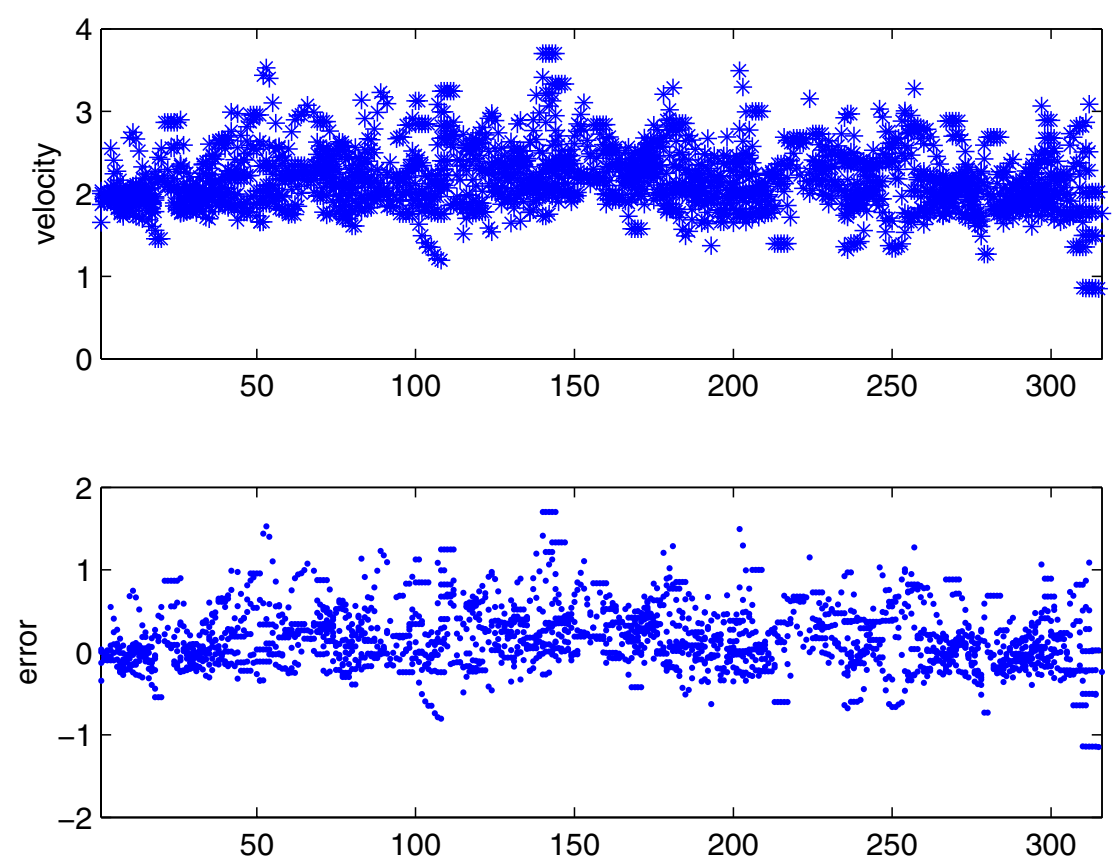

Fig. 6. Estimated magnitude velocity and corresponding errors for row number 5 to 55 for steps in 5 , i.e. 11 rows, at frame number 7

The horizon is broken at two places, approximately columns 0 - 30 and 170 200 , where the motion is to the left under the horizon. Accurate estimation of these velocities are not trivial. The result from estimation of the velocity for row number 20 is shown in Figure 5. The average error for this line is 0.25 pels when the correct velocity is 2.0 pels. The standard deviation is 0.19 . The density is $67 \%$. This corresponds to an average angular error less than 4 degrees when the absolute angular error is less than 4 degrees (see Figure 2). If the absolute angular error is approximately zero the average angular error is 2 degrees. The result for 11 rows covering the complete sky region is shown in Figure 6. It can be seen from this figure that there is a bias towards higher values for the magnitude velocity. The total average angular error is 0.3 with density $63 \%$. This also corresponds to less than 4 degrees average angular error for small absolute angular errors. The accuracy is in the same range as the best reported results except for Brox et al. and Bab-Hadiashar and Suter (see Figure 3, left). This means that we have been able to detect and measure the velocity in the sky region despite the fact that the simulation of the clouds have been based on fractal patterns. The real problems come when we want to estimate the velocity for positions at the horizon. Here we have multiple motions as demonstrated in Figure 4, right. This is a much tougher problem than what 
the clouds represents. We will not give any results on this, but just confirm that the challenge for the motion estimation algorithm is in this region and not in the sky.

\section{Discussion}

When we want to evaluate a method we should use realistic data. From our opinion the translating/diverging tree sequence and the Yosemite sequence without clouds are not sufficient to make a proper evaluation of a technique for estimation of optical flow. By excluding the sky region the occlusion boundary represented by the horizon is simplified. There will be a step between the velocities below the horizon and the zero velocity in the sky region, but this is much easier than the opposing velocities at the horizon when clouds move to the right.

We suggest to replace the Yosemite sequence with a new test sequence that includes occlusion boundaries and multiple motions. The Yosemite sequence could be modified by replacing the clouds with some flying objects (plane(s), balloon(s),etc.) with known velocity.

It is insufficient to publish the average angular error. We would like to know the distribution between angular and magnitude errors. In addition histograms of the errors, both magnitude and angular, will give a more complete picture of the performance for the actual algorithms.

To make a differentiation between different techniques it is also of interest to know what kind of structures or motion patterns that cause the largest errors.

\section{Conclusions and Future Work}

As we have argued in this paper, the problem is not the clouds, but it is mainly the occluding boundary with multiple motions. This challenge is reduced appreciable by removing the clouds such that the motion vectors in the sky region is zero. Our suggestion is to introduce flying objects in the sky instead. This will give a more realistic situation for test of the optical flow methods.

More work on error analysis is needed. This could be done in a manner as suggested in [23]. The performance at occlusion boundaries and more challenging motion patterns should be described in more detail for each algorithm.

In addition new and more challenging test sequences should be developed.

\section{Call for New Test Sequences!}

We will continue our work on estimation of optical flow and try to develop some new test sequences. We also calls for (thanks to the reviewer for this idea of a call for ...) suggestions from other workers in the field of motion estimation to suggest better test sequences and evaluation methods. 


\section{References}

1. Gibson, J.J.: The Perception of the Visual World. Houghton-Mifflin: Boston (1950)

2. Horn, B., Schunck, B.: Determining optical flow. Artificial Intelligence 17 (1981) $185-203$

3. Lucas, B., Kanade, T.: An iterative image registration technique with an application to stereo vision. In: Proc. DARPA Image Understanding Workshop. (1981) $121-130$

4. Limb, J.O., Murphy, J.A.: Estimating the velocity of moving images in television signals. Computer Graphics and Image Processing 4 (1975) 311-327

5. Barron, J., Fleet, D., Beauchemin, S., Burkitt, T.: Performance of optical flow techniques. Technical Report TR 299, Dept. Comp. Sci., Univ. Western Ontario (1993)

6. Barron, J., Fleet, D., Beauchemin, S.: Performance of optical flow techniques. International Journal of Computer Vision 12 (1994) 43-77

7. Black, M.J.: Frequently asked questions. Technical report, Department of Computer Science, Brown University, http://www.cs.brown.edu/people/black/ (2005)

8. Ngo, C.W., Pong, T.C., Zhang, H.J.: Motion analysis and segmentation through spatio-temporal slices processing. IEEE Trans. Image Processing 12 (2003) 341355

9. Knutsson, H., Andersson, M.: Loglets: Generalized quadrature and phase for local spatio-temporal structure estimation. In: Proceedings of the Scandinavian Conference on Image Analysis, SCIA 2003, Springer- Verlag (2003) 741-748

10. Austvoll, I.: Directional filters and a new structure for estimation of optical flow. In: Proc. Int. Conf. Image Proc. Volume II., IEEE Signal Processing Soc. Los Alamitos, CA, USA (2000) 574-577

11. Austvoll, I.: Motion Estimation using Directional Filters. PhD thesis, NTNU/HIS, PoBox 2557 Ullandhaug, N-4091 Stavanger, Norway. (1999)

12. Pauwels, E.J., Van Gool, L.J., Fiddelaers, P., Moons, T.: An extended class of scale-invariant and recursive scale space filters. IEEE Trans. Pattern Anal. and Machine Intell. 17 (1995) 691-701

13. Fleet, D., Jepson, A.: Computation of component image velocity from local phase information. International Journal of Computer Vision 5 (1990) 77-104

14. Foroosh, H.: Pixelwise-adaptive blind optical flow assuming nonstationary statistics. IEEE Trans. Image Processing 14 (2005) 222-230

15. Brox, T., Bruhn, A., Papenberg, N., Joachim, W.: High accuracy optical flow estimation based on a theory for warping. In: Proc. European Conference on Computer Vision, ECCV 2004, Springer- Verlag (2004) 25-36

16. Bab-Hadiashar, A., Suter, D.: Robust optical flow computation. Int. J. of Computer Vision 29 (1998) 59-77

17. Liu, H., Chellappa, R., Rosenfeld, A.: Accurate dense optical flow estimation using adaptive structure tensors and a parametric model. IEEE Trans. Image Processing 12 (2003) 1170-1180

18. Farnebäck, G.: Very high accuracy velocity estimation using orientation tensors, parametric motion, and simultaneous segmentation of the motion field. In: Proc. Int. Conf. Comp. Vis. Volume I. (2001) 171-177

19. Mémin, E., Pérez, P.: Dense estimation and object-based segmentation of the optical flow with robust techniques. IEEE Trans. Image Processing 7 (1998) 703 719 
20. Ju, S., Black, M.J., Jepson, A.D.: Skin and bones: Multi-layer, locally affine, optical flow and regularization with transparency. In: IEEE Conf. on Computer Vision and Pattern Recognition,CVPR'96. Volume 2182., San Francisco, CA (1996) 307-314

21. Chen, L.F., Liao, H.Y.M., Lin, J.C.: Wavelet-based optical flow estimation. IEEE Trans. Circuits and Systems for Video Tech. 12 (2002) 1-12

22. Granlund, G.H., Knutsson, H.: Signal Processing for Computer Vision. Kluwer Academic Publishers (1995)

23. Robinson, D., Milanfar, P.: Fundamental performance limits in image registration. IEEE Trans. Image Processing 13 (2004) 1185-1199 